\title{
GEO-INFORMATION TECHNOLOGIES FOR A MULTIMODAL ACCESS ON HISTORICAL PHOTOGRAPHS AND MAPS FOR RESEARCH AND COMMUNICATION IN URBAN HISTORY
}

\author{
F. Maiwald ${ }^{1}$, F. Henze ${ }^{1}$, J. Bruschke ${ }^{2}$, F. Niebling ${ }^{2}$ \\ ${ }^{1}$ Institute of Photogrammetry and Remote Sensing, TU Dresden, Germany - (ferdinand.maiwald, frank.henze)@ tu-dresden.de \\ ${ }^{2}$ Human-Computer Interaction, Julius-Maximilians-Universität Würzburg, Germany - \\ (jonas.bruschke, florian.niebling)@uni-wuerzburg.de
}

Commission II, WG II/8

KEY WORDS: Historical Photographs and Maps, Urban History, 3D Geo-Information System, Photogrammetry, Augmented Reality

\begin{abstract}
:
This contribution shows ongoing interdisciplinary research of the project HistStadt4D, concerning the investigation and development of different multimodal access strategies on large image repositories. The first part of the presented research introduces different methods of access, where classical analogue access stands in contrast to digital access strategies such as online collections, Web3D, Augmented Reality (AR) and Virtual Reality (VR). We discuss the main persisting issues of libraries, advantages of digital methods, and different access tools. The second part shows technologies and workflows used to create various access possibilities. The photogrammetric and geo-informational work serves as a technical basis for a 3D WebGIS as well as multiple AR/VR applications, which require spatial oriented images, object coordinates, and further spatial data. We introduce a research environment that allows art historians spatial access to historical photography, integrating 3D/4D models with photographic documents of the respective architecture. For dissemination of research results in installations and museums, we present fully immersive VR as well as handheld AR applications allowing users a free exploration of historical photography in a spatial setting.
\end{abstract}

\section{INTRODUCTION}

The access to extensive repositories of historical photographs and maps using classical database systems presupposes a comprehensive knowledge about structure and semantics of the respective database objects and is aimed above all at experienced users. Without knowledge of the depicted building objects as well as the concrete structuring of data, like keywords, ontologies and metadata, search queries often do not lead to the desired results.

The project HistStadt4D aims to investigate and prototypically develop access to such repositories via a spatio-temporal location of historical photographs and maps within a 3D model of the city center of Dresden/Germany as well as to assess usage options and cultural implications. The project consists of 9 researchers with various disciplinary backgrounds such as information behavior, education, architectural history, photogrammetry and computer science. The central component is a web-based 4D browser, which takes into account temporal information in addition to the 3D spatial information on the media and building objects. The spatial visualization of location-based media and objects via a 3D city model is intended to enable an extended circle of users accessing urban history information as simply and intuitively as possible. A tackled scenario is to provide a spatio-temporal scaled interface for large-scale collections of urban images. If all objects are located in a higher-level coordinate reference system, the $3 \mathrm{D}$ model should serve as the basis for a location-dependent AR representation on mobile devices. Among other scenarios it can be used to support city tourism and location-based education about urban history.

The first step when working in large data repositories is the extraction of relevant data for prototype development. The image data has to be filtered considering the advantage when spatialized in a $3 \mathrm{D}$ environment. Content-based image retrieval using neural networks are a first step of filtering, e.g. images of buildings which, in the following, can be placed in the web application.

Then, these images can be spatially oriented using photogrammetric methods. Different approaches when working with historical data are presented. Interactive direct linear transformation (DLT), spatial resection or completely manual placing of the images in the 3D space were tested in the prototype application. Subsequently, the development and first experiences with the web-based 4D browser as the central user interface for the access to media repositories will be discussed. Different strategies showing the images and the $3 \mathrm{D}$ models as a $4 \mathrm{D}$ application have been developed. Using spatial projection, it can be determined which image depicts which object geometry. As this linkage is stored, the $3 \mathrm{D}$ objects can be used for interaction as an additional way to filter the images next to conventional search filters. The platform also provides tools for expert users, i.e. art and architectural historians, to support answering specific research questions. Backed by spatial information, diverse visualization methods enable investigation of, e.g., the statistical distribution of images in respect to their orientation or the depicted objects.

The junior research group aims to provide tools for Cultural Heritage education in different settings using Augmented Reality. The focus is on the communication of research results concerning historical photography of architecture to the general public through exhibitions, as well as guided and unguided tours. Bringing geo-located photography and historic models into the current cityscape through hand-held AR, tourists are enabled to engage with the historic situation of the photographer. In addition 
to location-based AR, we develop tools for the exploration of large amounts of photographic documents in exhibitions, combining 3D printed models of architecture with geo-located photographs in hand-held as well as see-through AR settings. Using the computed spatial orientation of photographs with respect to the respective 3D models of buildings, 3D-printed models can be augmented with historical images to provide historical textures. The article aims to provide an overview of current research and development work in the ongoing interdisciplinary research project HistStadt4D. It shows how multimodal access on image repositories has been realized in the past and how it will be implemented in the future. The workflow from classical repositories as databases over filtering and spatializing the images to presentation and interactive browsing in a web application as well as in an AR environment are presented.

\section{MULTIMODAL ACCESS ON IMAGE REPOSITORIES}

There are a lot of different ways to get access to multimodal data resources like plans, maps, images, etc., from different points in time. This contribution structures access strategies in four categories and explains how this kind of access is tackled in the project HistStadt4D. Classical interactive access to repositories with the requirement of being on site stands in contrast to the virtual access using online collections, web applications and technologies like Augmented Reality (AR) and Virtual Reality (VR).

\subsection{Classical access}

When working with very specific historical data, it is still often necessary to crawl archives and find source documents piece by piece, especially, since neither the digitization nor the annotation of huge data archives is fully completed. Four main issues that still persist in libraries and archives have e.g. been identified by Evens and Hauttekeete as (Evens and Hauttekeete, 2011):

1. the complex process of digitization

2. identification of correct metadata

3. business models/financing

4. intellectual property rights management

Thus, even in recent research, it is often necessary to collect analogue data and process it for the desired purpose. In the following, some examples in the field of Geomatics and Restoration are presented. Ackermann and Glekas modelled the Larz Anderson Estate using mainly photographs, journals, and drawings scattered in several American archives (Ackerman and Glekas, 2017). Also, Grussenmeyer and Khalil were able to reconstruct the Great Mosque of Aleppo with image data on 16 CD-ROMs using Structure from Motion (SfM) (Grussenmeyer and Al Khalil, 2017) while recently Bevilacqua et al. recreated parts of the Fortezza Vecchia using archive images and plans from different sources (Bevilacqua et al., 2019). Consequently, the classical process of archive work from exploring and finding data to accessing and digitizing sources is still present nowadays. The presented project does not heavily rely on classical access because of the existing cooperation to online media repositories. Data that is not yet digitized is not the main interest since the goal is enhancing and creating digital tools for the multimodal access on image repositories.

\subsection{Virtual access using online collections}

As already stated, digital information is and will be essential for automated workflows as well as the dissemination of information and knowledge in society. Ross and Hedstrom therefore proposed six reasons why digital preservation plays an important role in cultural heritage institutions. These are in brief (Ross and Hedstrom, 2005):

1. protection and conservation of cultural memory is a societal good

2. international scientific collaborations benefit from the availability of data repositories

3. accountability of those institutions

4. re-use of digital information as economic benefit

5. effective and affordable strategies as move from an industrial to a knowledge economy

6. sustainable digital libraries depend upon the availability of preservation tool and services

When looking at today's archives, data is usually presented in one or two dimensions. Hence, objects, books, photographies, and other information is displayed as text or can be depicted in image form. Examples for comprehensive online collections are Europeana (europeana.eu), Artstor (artstor.org) and arachne (arachne.dainst.org) and there exist many more. Usually the websites providing access to these repositories show a search bar in which users can type keywords (fig. 1). Those lead to a

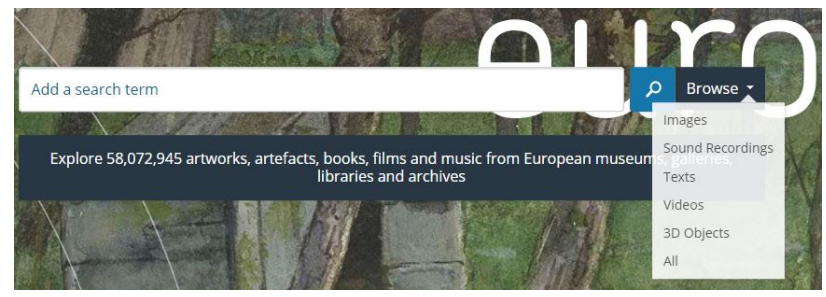

Figure 1. Search bar of europeana.de showing additional filter options

resulting page where numerous filters (e.g. media classification, contributor, license, etc.) can be applied. Nonetheless, challenges such as a low data quality, difficult access, usability problems and poor availability of information in certain areas of interest still persist today (Münster et al., 2018). Considering the search bar, metadata plays an important role in finding the relevant object or data file but also limits the search possibilities. Problems arise when the metadata is missing, incorrect, or insufficient. Then, it will not be possible for a user to find the specific object. Filters can help to narrow the search query but are often not enough to simplify the results. Additionally, different types of users are following diverse search strategies and for most of the users, Google is still the main entrance for search queries (Beaudoin and Brady, 2011). It has been investigated by different research groups that a beginner uses far more simple terms than an expert for metadata-based search queries and that online data retrieval and access is still a challenge for many people (Beaudoin and Brady, 2011, Fleming-May and Green, 2016, Münster et al., 2018, Yoon and Chung, 2011). In HistStadt4D, access to historical images is granted via the photo library of the Saxon State and University Library Dresden (deutschefotothek.de) where similar issues are present which 
are improved during the project. In addition, the mentioned repository holds maps, drawings and scaled plans which could be added later as further geospatial information source.

\subsection{D/4D access using web applications}

Since the contribution presents the steps towards a 3D and 4D (3D models + time dimension) environment for presenting data, the advantages of such models and ongoing research in that field is presented. 3D web browsers in cultural heritage are also referred to as Web3D exhibitions (Styliani et al., 2009). A three-dimensional representation of an object provides most often an improvement in understanding. Proportions, i.e. the real size of objects, can be visualized better in 3D space because of the possibility of comparing them to other objects in an additional dimension (Patera, 2009). Advantages for various user scenarios that have been investigated in several studies (Dorta and Pérez, 2006, Okeil, 2010, Patera, 2009) are:

- Better and enhanced understanding of objects

- Advanced detection of broken/damaged objects

- Exciting learning activity combined with a lower level of frustration

- Enhanced spatial awareness including the scale of objects

- Improved communication about 3D objects

- (Depending on the application) a powerful set of creation and manipulation tools

Objects that benefit heavily from a visualization in 3D space are exhibits of museums like e.g. coins, pottery, or bones. Additionally, architecture and historical buildings can be explored in greater detail by users. It has to be said that a three-dimensional representation of any object is not always necessary, but often provides a better understanding (Heine and Henze, 2009).

Web3D examples where different objects are shown are CultLab3D (cultlab3d.de) (fig. 2), the official Web3D website (web3d.org), and x3dom (x3dom.org).

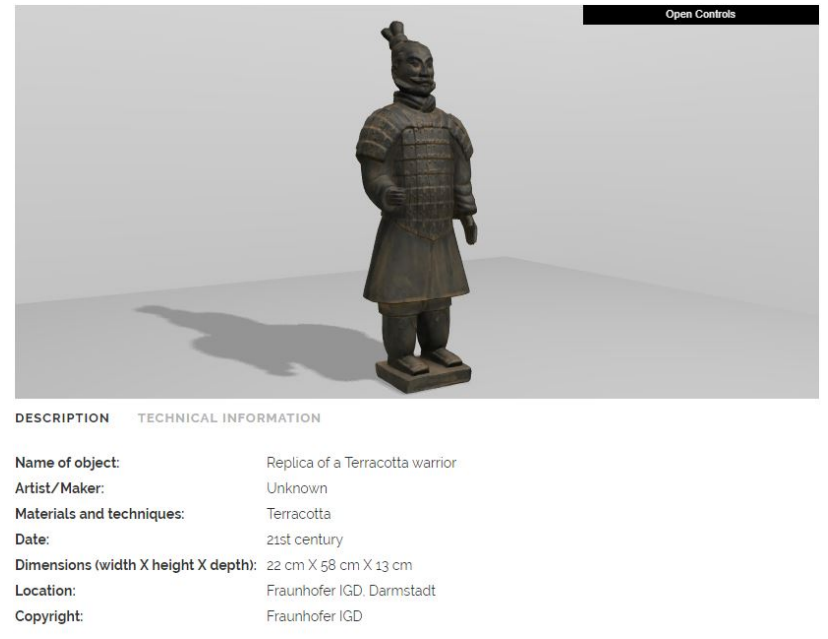

Figure 2. Replica of a Terracotta warrior and additional metadata presented in a Web3D browser environment (cultlab3d.de)

The web application of HistStadt4D showing a three-dimensional city model of Dresden in addition to oriented historical photographies in a spatial and temporal domain can be accessed at http://4dbrowser .urbanhistory4d.org

\subsection{Augmented Reality and Virtual Reality access}

Advantages described in the previous section also largely apply to AR and VR access. Several researchers discovered that objects shown in AR/VR are easier to understand and provide enhanced methods of user interaction (Styliani et al., 2009, Bekele et al., 2018). Carrozzino classified different access methods in two categories of immersion and interaction and found out that current research works on less expensive, less complex, more easily and cheaply sustainable devices (Carrozzino and Bergamasco, 2010).

But not only applications in cultural heritage profit from this access method. Further applications are in the fields of tourism, psychology, industry, medicine, education, and research. In opposite to 3D web applications which are usually accessed using PCs, tablets or smartphones - for AR/VR - access strategies can be realized using stationary as well as wearable computer systems, e.g. head mounted displays (HMD), and mobile devices (Rigby and Smith, 2013).

Especially for cultural heritage, Slater and Sanchez-Vives identified four advantages and accompanying applications as follows (Slater and Sanchez-Vives, 2016):

1. Worldwide access and exploration of cultural sites

2. Preservation by digitization of cultural sites

3. Restoration and experience of cultural sites

4. Modeling of cultural sites under different future conditions

In HistStadt4D models and photographic images are combined in virtual worlds, using purely virtual models in VR, as well as physical 3D-printed models in AR installations (Niebling et al., 2018a). In both VR and AR, historical images are projected onto 3D models dynamically, providing historical texturing to enable users to re-experience the environment of the photographer at the time the respective photos were taken (Niebling et al., 2018b).

\section{WORKFLOW TOWARDS FOURDIMENSIONAL MODELS}

The project HistStadt4D focuses especially on image repositories and how access for different users can be granted. The research group mainly works with historical photographs showing architecture and additional 3D models of buildings. In the following, the workflow from a single image to the $4 \mathrm{D}$ application is shown. Starting point are multiple photogrammetric approaches for image selection and image orientation. Afterwards, the processed images are placed in the web environment and the orientations serve for different applications in the field of Augmented and Virtual Reality.

\subsection{Photogrammetric approaches as a technical basis}

The first necessary step aiming at placing images in a spatial domain requires a precise choice of suitable images. Since image repositories do not exclusively show photographies, the relevant data has to be filtered in a first step. The simplest approach is using metadata search for a specific building and narrowing the results using e.g. the "photography" filter. A look at the remaining images shows that there are still a number of irrelevant hits like e.g. interior of the building, photographs taken from the top of the building, images from events only linked by metadata to the respective building (Niebling et al., 2017). 


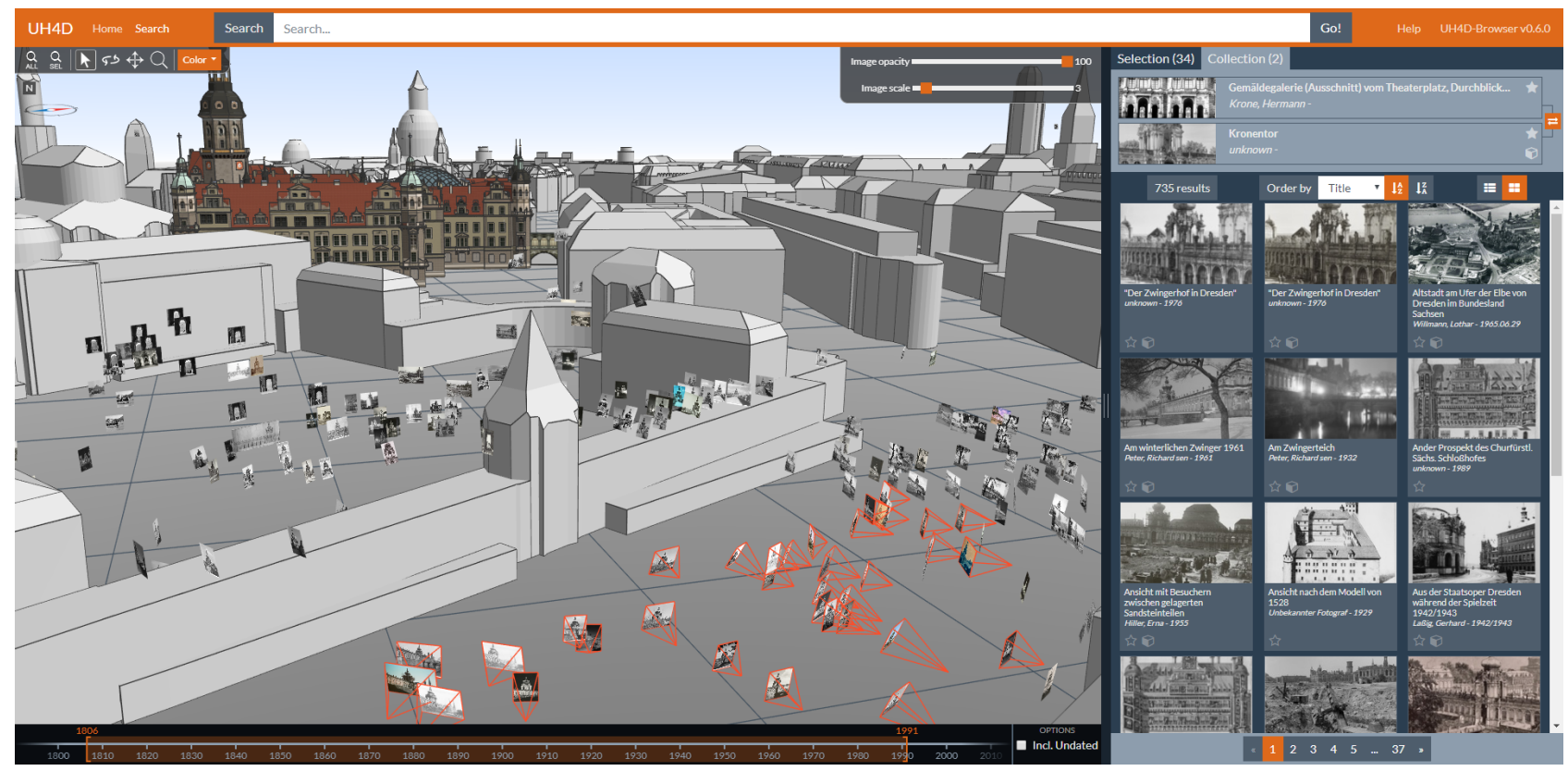

Figure 3. Prototype browser application of HistStadt4D with interactively and automatically oriented images

This leads to the question how to get only desired data required for photogrammetric processing. The metadata-based search query could be extended by a subsequent image-based search. E.g. Europeana already shows similar objects as suggested content for one image. In HistStadt4D, studies have been performed filtering images using content-based image-retrieval with support vector machines and additionally deep learning approaches. In cooperation with ScaDS (scads.de), a filtering of historical images was shown to be possible for a small sample using the convolutional network VGG16 (Simonyan and Zisserman, 2014) in a transfer learning approach. Still, it is not yet possible to get exclusively relevant results, so further approaches have to be tested.

In the next step, the filtered images have to be oriented in 3D space. When the building still exists and 3D data is available, an interactive direct linear transformation can be calculated to estimate the inner and exterior orientation of the camera followed by a spatial resection approach (Bruschke et al., 2018a) (fig. 3). There also exist fully automatic approaches for the registration of 3D object and 2D image data (Boerner and Kröhnert, 2016, Kehl et al., 2017).

If there is no object information, calculation of the orientation is significantly harder. The focus in the project lies on the determination of a relative orientation between the historical images. Subsequently, the geometry of 3D structures can be calculated using the known or estimated inner orientation of the camera. The relative orientation of image pairs in a projective space can be determined using homologue points in both images. This can be a quite challenging task for historical images due to digitization artifacts, different image mediums, cameras and acquisition techniques (Maiwald, t.b.p. 2019).

Thus, various methods have been tested, e.g. the combination of historical and recent images for orientation (Maiwald et al., 2017), feature matching using the geometry of quadrilaterals (Maiwald et al., 2018) and special feature matching methods for difficult image pairs such as RIFT (Li et al., 2018), MSER (Matas et al., 2004) and MODS (Mishkin et al., 2015). The latter

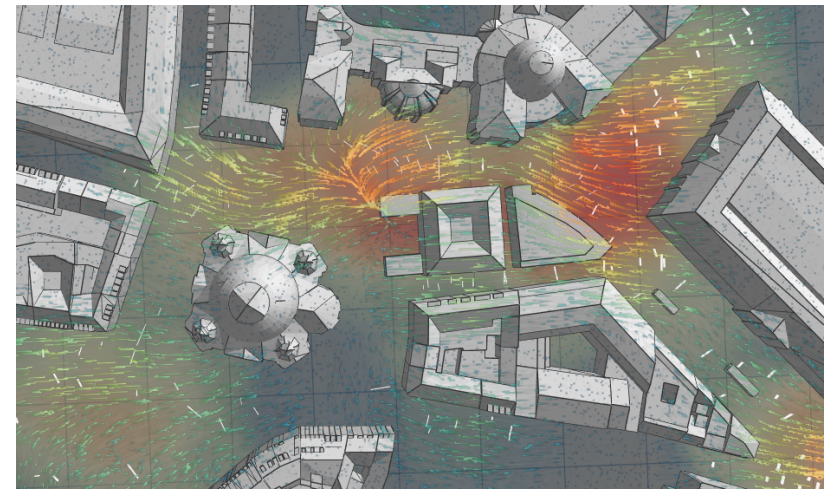

Figure 4. Statistical visualizations of images taking their positions and orientations into account

approach showed the best results and in the future it is planned to orient multiple historical images using those methods in a bundle adjustment. Image sizes, orientation data (coordinates in a global frame), camera angles and focal length can then be exported to further applications.

It may be possible in the future that additional geo-informational data such as plans, maps, BIMs can be added to the 4D browser environment. Still, due to ambiguous historical data and a large variety in Geographical Information standards, integration in such systems is difficult (Previtali and Latre, 2018).

\subsection{Browser application}

The current browser-based prototype (4dbrowser. urbanhistory4d.org) employs a search bar for entering keywords and a results list known from conventional front ends to image databases. These basic components are enhanced by a $3 \mathrm{D}$ viewport that forms the main part of the graphical user interface (fig. 3). Utilizing a contemporary 3D city model, the images are displayed in their spatial context.

This enhances the understanding of the position of the photographer, i.e. from where the photograph has been taken 

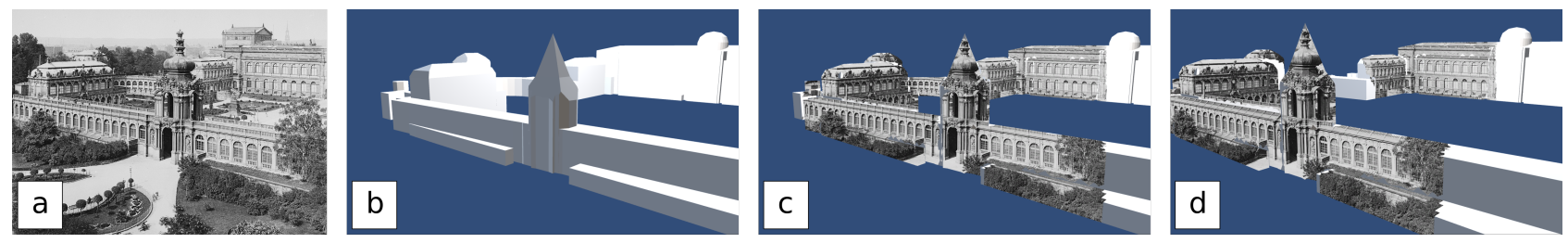

Figure 5. (a) Historic depiction of Dresdner Zwinger. (b) LOD2 CAD model provided by the city municipality. (c) Projection of image to model and usage as texture. (d) Changing viewpoint with applied texture, structures hidden in the original image are left blank.

(Schindler and Dellaert, 2012). The user is able to take up the photographer's position and blend the image with the 3D model to detect changes in construction (Bruschke et al., 2017). An interactive time slider is available that enables the user to filter the images by specific time spans. In addition, the images can be filtered by buildings they are depicting by selecting the corresponding 3D objects. In regard to the usage by experts such as art and architectural historians, advanced statistical visualization methods can be applied to the images to support answering specific research questions (Bruschke et al., 2018b). This includes conventional heat maps but also visualizations that consider the orientation of the images (fig. 4).

The browser application utilizes AngularJS as a basic front end web framework to build single-page applications (SPA). Regarding 3D graphics, three.js is used as a higher level API for WebGL. Owing the SPA approach, all loaded 3D content is kept in the background when the user switches to another subpage. When switching back to the main interactive 3D view, the $3 \mathrm{D}$ content has not to be loaded again and can be displayed instantly. The back end is a REST API on the basis of Node.js and Express.js. It handles not only all database requests, but also routines to automatically retrieve metadata from the original image repository. The data itself is stored compliant to the CIDOC Conceptual Reference Model (CRM). This results in strongly connected data that is predestined to be stored not in a relational database but in a graph database, in this case $N e o 4 j$ (Bruschke and Wacker, 2014).

\subsection{Augmented Reality / Virtual Reality}

HMD-based fully immersive VR as well as handheld AR are employed to allow users free exploration of historical photography in a spatial setting. We combine 3D models of architecture with geo-located photography to elicit spatial awareness concerning positions and also orientations of photographers during shooting of photos. For user engagement, we create gamification elements in VR, e.g. competitively locating positions from where photos were taken (fig. 6).

Here, users are challenged to navigate to specific locations in a full-scale 3D model of the Dresdner Zwinger, and try to replicate given iconic depictions of historical settings both faster and more accurate than their opponents.

We create historic textures for registered models of architecture by UV mapping vertices of the respective $3 \mathrm{D}$ model to the historic photography in both Virtual and Augmented Reality. UV coordinates are calculated by casting rays from the position $P$ of the photographer to each vertex $V$ in the model, through the projective plane of the currently processed image $I$.

Collision detection of rays with models in the scene is performed to detect visibility of vertices from position $P$. Parts of a building
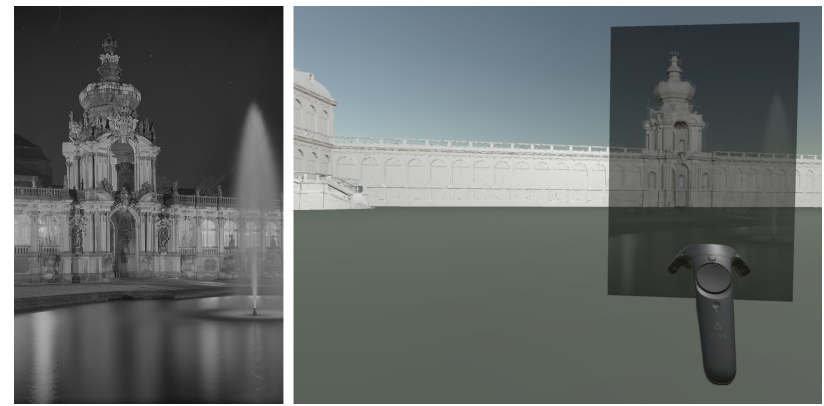

Figure 6. HMD-based VR game to find location and orientation of photographer. Right: Semi-transparent VR overlay

that are not contained in $I$, because they are either outside of the view frustum of the camera (cf. fig. 5c) or hidden by other structures (cf. fig. 5d), cannot be textured with information contained within $I$ and are left blank. Employing photos as textures allows for the usage of very coarse 3D models of buildings, as details are provided by comparably high resolution photos.

For exploration of historical photography in a model-sized environment, we combine the above projection method with physical models. 3D models procured from the city municipality are 3D-printed into physical models to be used in a hand-held video see-through AR setup based on a 10.1 inch Android tablet. (fig. 7). Tracking of the model is provided by Vuforia Model Targets with Advanced Recognition in a Unity application, combining the digital model of the building with a media repository of spatially registered historical photography.

We render the textured digital model on top of the video of the tracked physical model, discarding parts that are not contained in the respective photo selected by the user. Spatial movement of the tablet can be performed to view buildings from a perspective diverging from the perspective of the photo, but still being able to perceive the historical appearance of the building depicted in the historical image. The tablet can then be used to access and browse the catalogue of images in AR, allowing selection of photography according to the positions of the spatially registered documents.

\section{CONCLUSIONS AND OUTLOOK}

The contribution presents different access methods on historical data. It is shown that classical analogue access is still important for multiple current research workflows due to different issues persisting in classical libraries. Still, digital methods are growing e.g. in the field of cultural heritage since they provide various advantages. Virtual libraries usually grant users access to data wherever they are and whenever they want. Additonally, it has 


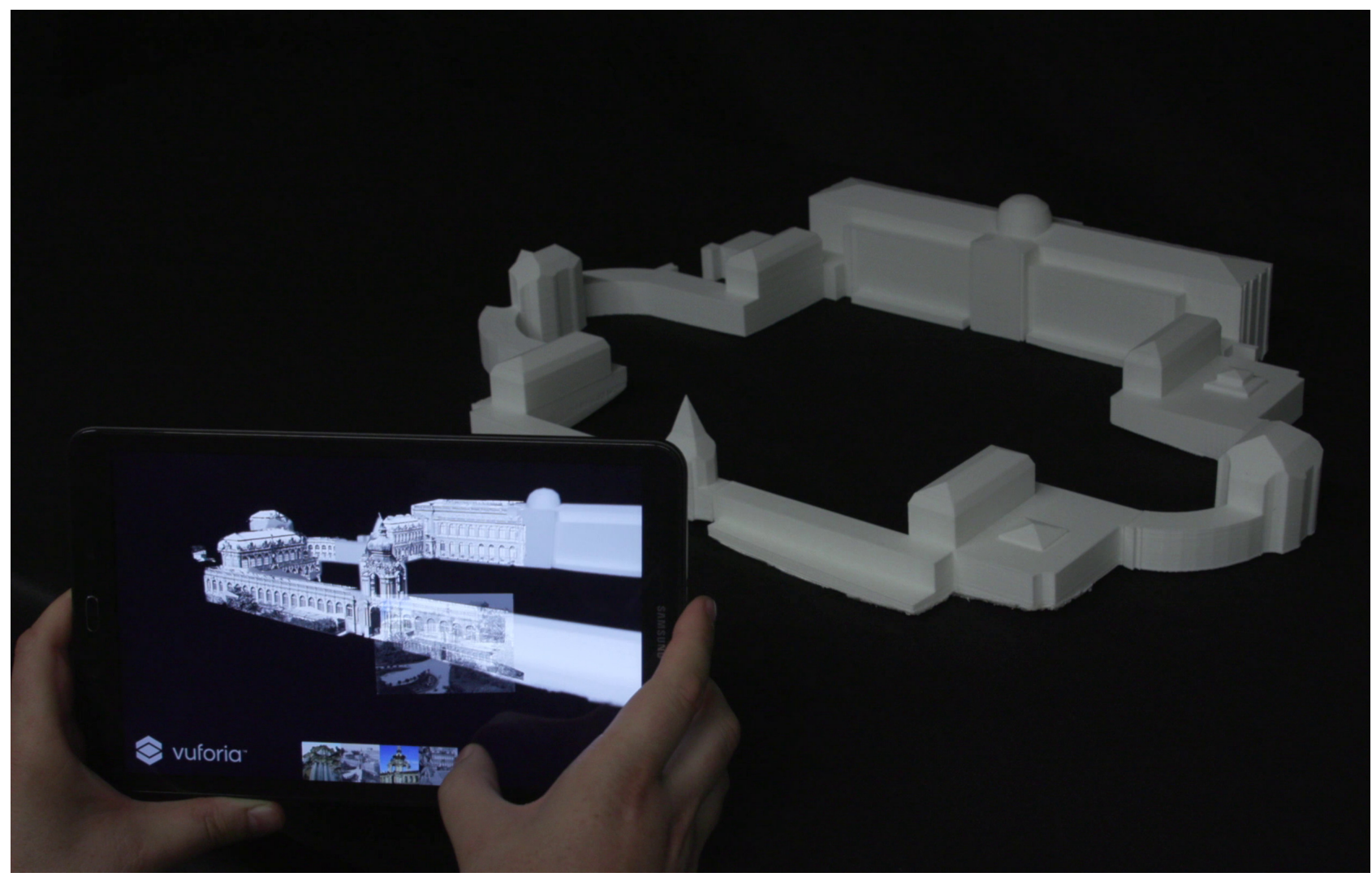

Figure 7. Augmented Reality installation using historical photography as textures for 3d-printed model of architecture

been investigated that $3 \mathrm{D}$ repositories enhance the understanding of objects and improve possibilities of communication.

The project HistStadt4D realizes a virtual repository using different techniques. Geoinformation data is spatialized using geodetic and photogrammetric methods. The focus lies especially on the relative orientation of images and in the following, the orientation in a global frame which is relevant for the web application and different AR/VR implementations. The prototype of the $4 \mathrm{D}$ browser uses a classical search bar combined with a $3 \mathrm{D}$ viewport showing these spatialized buildings and historical images. A time slider allows the filtering of the images and thus, adds the temporal component as the fourth dimension. For further research purposes different statistical visualizations of image orientations can be displayed.

The AR/VR implementations allow users to explore the geoinformation data in a spatial setting. The combination of 3D-printed models and see-through AR could serve as a information base in a museum as the projection of images creates immersive scenes. Gamification methods are realized in VR to enhance the orientation of images and test different competitive concepts.

In the future, it would be desirable to connect the different parts more closely and automatically in a single workflow. As a starting point, the images are digitized by a classical library and provided with metadata. Using the metadata the images could be categorized and automatically oriented using the already existing orientations. Then, the images including the intrinsic and extrinsic camera parameters are directly transferred to the applications. After that, the user gets a notification that new data is available for research purposes.
Our aim is to realize such a workflow and find the best strategy to present geospatial data to a broad community.

\section{ACKNOWLEDGEMENTS}

The research upon which this paper is based is part of the junior research group UrbanHistory4D's activities which has received funding from the German Federal Ministry of Education and Research under grant agreement No 01UG1630.

\section{REFERENCES}

Ackerman, A. and Glekas, E., 2017. Digital capture and fabrication tools for interpretation of historic sites. ISPRS Annals of Photogrammetry, Remote Sensing and Spatial Information Sciences IV-2/W2, pp. 107-114.

Beaudoin, J. E. and Brady, J. E., 2011. Finding visual information: a study of image resources used by archaeologists, architects, art historians, and artists. Art Documentation: Journal of the Art Libraries Society of North America 30(2), pp. 24-36.

Bekele, M. K., Pierdicca, R., Frontoni, E., Malinverni, E. S. and Gain, J., 2018. A survey of augmented, virtual, and mixed reality for cultural heritage. Journal on Computing and Cultural Heritage (JOCCH) 11(2), pp. 7.

Bevilacqua, M., Caroti, G., Piemonte, A. and Ulivieri, D., 2019. Reconstruction of lost architectural volumes by integration of photogrammetry from archive imagery with 3-d models of the status quo.

Boerner, R. and Kröhnert, M., 2016. Brute force matching between camera shots and synthetic images from point clouds. ISPRS - International Archives of the Photogrammetry, Remote Sensing and Spatial Information Sciences XLI-B5, pp. 771-777. 
Bruschke, J. and Wacker, M., 2014. Application of a graph database and graphical user interface for the cidoc crm. In: Access and Understanding-Networking in the Digital Era. Session J1. The 2014 annual conference of CIDOC, the International Committee for Documentation of ICOM.

Bruschke, J., Maiwald, F., Münster, S. and Niebling, F., 2018a. Browsing and experiencing repositories of spatially oriented historic photographic images. SDH 2(2), pp. 138-149.

Bruschke, J., Niebling, F. and Wacker, M., 2018b. Visualization of orientations of spatial historical photographs. In: R. Sablatnig and M. Wimmer (eds), Eurographics Workshop on Graphics and Cultural Heritage, The Eurographics Association, pp. 189-192.

Bruschke, J., Niebling, F., Maiwald, F., Friedrichs, K., Wacker, M. and Latoschik, M. E., 2017. Towards browsing repositories of spatially oriented historic photographic images in $3 \mathrm{~d}$ web environments. In: Proceedings of the 22nd International Conference on 3D Web Technology, ACM, p. 18.

Carrozzino, M. and Bergamasco, M., 2010. Beyond virtual museums: Experiencing immersive virtual reality in real museums. Journal of Cultural Heritage 11(4), pp. 452-458.

Dorta, T. and Pérez, E., 2006. Immersive drafted virtual reality a new approach for ideation within virtual reality. Proceedings of Synthetic Landscapes ACADIA 2006 pp. 304-316.

Evens, T. and Hauttekeete, L., 2011. Challenges of digital preservation for cultural heritage institutions. Journal of Librarianship and Information Science 43(3), pp. 157-165.

Fleming-May, R. A. and Green, H., 2016. Digital innovations in poetry: Practices of creative writing faculty in online literary publishing. Journal of the Association for Information Science and Technology 67(4), pp. 859-873.

Grussenmeyer, P. and Al Khalil, O., 2017. From metric image archives to point cloud reconstruction: Case study of the great mosque of aleppo in syria. ISPRS - International Archives of the Photogrammetry, Remote Sensing and Spatial Information Sciences XLII-2/W5, pp. 295-301.

Heine, K. and Henze, F., 2009. CISAR-ein modulares Informationssystem für raumbezogene Daten aus Archäologie und Bauforschung. Denkmäler3.de - Industriearchäologie, Tagungsband des interdisziplinären Kolloquiums vom 5.-7. November 2008 in Essen pp. 83-90.

Kehl, C., Buckley, S. J., Viseur, S., Gawthorpe, R. L. and Howell, J. A., 2017. Automatic illumination-invariant image-to-geometry registration in outdoor environments. The Photogrammetric Record 32(158), pp. 93-118.

Li, J., Hu, Q. and Ai, M., 2018. Rift: Multi-modal image matching based on radiation-invariant feature transform. arXiv preprint arXiv: 1804.09493.

Maiwald, F., Schneider, D., Henze, F., Münster, S. and Niebling, F., 2018. Feature matching of historical images based on geometry of quadrilaterals. ISPRS - International Archives of the Photogrammetry, Remote Sensing and Spatial Information Sciences XLII-2, pp. 643-650.

Maiwald, F., Vietze, T., Schneider, D., Henze, F., Münster, S. and Niebling, F., 2017. Photogrammetric analysis of historical image repositories for virtual reconstruction in the field of digital humanities. The International Archives of Photogrammetry, Remote Sensing and Spatial Information Sciences 42, pp. 447.

Matas, J., Chum, O., Urban, M. and Pajdla, T., 2004. Robust wide-baseline stereo from maximally stable extremal regions. Image and vision computing 22(10), pp. 761-767.
Mishkin, D., Matas, J. and Perdoch, M., 2015. Mods: Fast and robust method for two-view matching. Computer Vision and Image Understanding 141, pp. 81-93.

Münster, S., Kamposiori, C., Friedrichs, K. and Kröber, C., 2018. Image libraries and their scholarly use in the field of art and architectural history. International Journal on Digital Libraries 19(4), pp. 367-383.

Niebling, F., Bruschke, J. and Latoschik, M. E., 2018a. Browsing Spatial Photography for Dissemination of Cultural Heritage Research Results using Augmented Models. In: R. Sablatnig and M. Wimmer (eds), Eurographics Workshop on Graphics and Cultural Heritage, The Eurographics Association.

Niebling, F., Maiwald, F., Barthel, K. and Latoschik, M. E., 2017. 4D Augmented City Models, Photogrammetric Creation and Dissemination. Digital Research and Education in Architectural Heritage, Springer International Publishing, Cham, pp. 196-212.

Niebling, F., Münster, S., Bruschke, J., Maiwald, F. and Friedrichs, K., 2018b. Stadtgeschichtliche Forschung anhand räumlich- und zeitlich verorteter Photographien. In: M. Burghardt and C. Müller-Birn (eds), INF-DH-2018, Gesellschaft für Informatik e.V., Bonn.

Okeil, A., 2010. Hybrid design environments: immersive and non-immersive architectural design. Journal of Information Technology in Construction (ITcon) 15(16), pp. 202-216.

Patera, M., 2009. The Potential of 3D Visualisation Technology in Art and Design Education. Thesis, University of Glasgow.

Previtali, M. and Latre, M. A., 2018. A brokered virtual hub approach for the generation of web applications based on historical maps. Applied Geomatics 10(4), pp. 453-472.

Rigby, J. and Smith, S. P., 2013. Augmented reality challenges for cultural heritage. Newcastle: Applied Informatics Research Group University of Newcastle.

Ross, S. and Hedstrom, M., 2005. Preservation research and sustainable digital libraries. International journal on digital libraries 5(4), pp. 317-324.

Schindler, G. and Dellaert, F., 2012. 4d cities: Analyzing, visualizing, and interacting with historical urban photo collections. Journal of Multimedia 7(2), pp. 124-131.

Simonyan, K. and Zisserman, A., 2014. Very deep convolutional networks for large-scale image recognition. arXiv preprint arXiv:1409.1556.

Slater, M. and Sanchez-Vives, M. V., 2016. Enhancing our lives with immersive virtual reality. Frontiers in Robotics and AI 3, pp. 74 .

Styliani, S., Fotis, L., Kostas, K. and Petros, P., 2009. Virtual museums, a survey and some issues for consideration. Journal of cultural Heritage 10(4), pp. 520-528.

Yoon, J. and Chung, E., 2011. Understanding image needs in daily life by analyzing questions in a social q\&a site. Journal of the American Society for Information Science and Technology 62(11), pp. 2201-2213. 